\title{
SCALING INVARIANT HARDY TYPE INEQUALITIES WITH NON-STANDARD REMAINDER TERMS
}

\author{
MEGUMi SANO
}

Abstract. We consider the Hardy inequality on $\mathbb{R}^{N}$, the critical Hardy inequality on a ball, and the Rellich inequality on $\mathbb{R}^{N}$. These three Hardy type inequalities can be refined by adding remainder terms. Our remainder terms are expressed by a distance from the families of "virtual" extremals. A key ingredient is the critical Hardy inequality on $\mathbb{R}^{N}$ which was proved by Machihara, Ozawa and Wadade [21].

Mathematics subject classification (2010): 35A23, 26D10.

Keywords and phrases: Hardy type inequality, remainder term, virtual extremal.

\section{REFERENCES}

[1] Adimurthi, N. Chaudhuri And M. Ramaswamy, An improved Hardy-Sobolev inequality and its application, Proc. Amer. Math. Soc. 130 (2002), no. 2, 489-505 (electronic).

[2] Adimurthi, M. Grossi, S. AND S ANTRA, Optimal Hardy-Sobolev inequalities, maximum principle and related eigenvalue problem, J. Funct. Anal. 240 (2006), 36-83.

[3] Adimurthi, K. SANDEeP, Existence and non-existence of the first eigenvalue of the perturbed Hardy-Sobolev operator, Proc. Roy. Soc. Edinburgh Sect. A 132 (2002), no. 5, 1021-1043.

[4] Adimurthi, S. Santra, Generalized Hardy-Rellich inequalities in critical dimension and its applications, Commun. Contemp. Math. 11 (2009), no. 3, 367-394.

[5] P. Baras, J. A. Goldstein, The heat equation with a singular potential, Trans. Amer. Math. Soc., 284 (1984), 121-139.

[6] E. BERCHIO, D. CASSANI, AND F. GAZZOLA, Hardy-Rellich inequalities with boundary remainder terms and applications, Manuscripta Math., 131 (2010), 427-458.

[7] H. BReZIS, J. L. VÁZQUEZ, Blow-up solutions of some nonlinear elliptic problems, Rev. Mat. Univ. Complut. Madrid 10 (1997), no. 2, 443-469.

[8] N. CHAUDHURI, M. RAMASWAMY, Existence of positive solutions of some semilinear elliptic equations with singular coefficients, Proc. Roy. Soc. Edinburgh Sect. A 131 (2001), no. 6, 1275-1295.

[9] A. CiAnchi, A. Ferone, Hardy inequalities with non-standard remainder terms, Ann. Inst. H. Poincaré. Anal. Nonlinéaire, 25, (2008), 889-906.

[10] E. B. DAVIES, A. M. HinZ, Explicit constants for Rellich inequalities in $L^{p}(\Omega)$, Math. Z. 227 (1998), no. 3, 511-523.

[11] A. Detalla, T. Horiuchi, H. Ando, Missing terms in Hardy-Sobolev inequalities, Proc. Japan Acad. Ser. A Math. Sci. 80 (2004), no. 8, 160-165.

[12] S. FilipPas, A. Tertikas, Optimizing improved Hardy inequalities, J. Funct. Anal. 192 (2002), $186-233$.

[13] F. Gazzola, H. C. GRUnau, E. Mitidieri, Hardy inequalities with optimal constants and remainder terms, Trans. Amer. Math. Soc. 356 (2003), no. 6, 2149-2168.

[14] F. Gazzola, H. C. Grunau, G. Sweers, Polyharmonic boundary value problems, LNM, vol. 1991, Springer, 2010.

[15] N. Ghoussoub, A. Moradifam, On the best possible remaining term in the Hardy inequality, Proc. Natl. Acad. Sci. USA. 105 (2008), no. 37, 13746-13751.

[16] N. Ghoussoub, A. Moradifam, Bessel pairs and optimal Hardy and Hardy-Rellich inequalities, Math. Ann. 349 (2011), no. 1, 1-57. 
[17] D. Gilbarg, N. S. Trudinger, Elliptic Partial Differential Equations of Second order (2nd ed.), Springer, New York, 1983.

[18] N. IOKU, M. ISHIWATA, A scale invariant form of a critical Hardy inequality, International Mathematics Research Notices, vol. 2015, no. 18, pp. 8830-8846.

[19] N. IOKU, M. Ishiwata, T. Ozawa, Sharp remainder of a critical Hardy inequality, Arch. Math. (Basel) 106 (2016), no. 1, 65-71.

[20] P. LindQvist, On the equation $\operatorname{div}\left(|\nabla u|^{p-2} \nabla u\right)+\lambda|u|^{p-2} u=0$, Proc. Amer. Math. Soc. 109 (1990), no. $1,157-164$.

[21] S. Machinara, T. Ozawa, H. Wadade, Scaling invariant Hardy inequalities of multiple logarithmic type on the whole space, J. Inequal. Appl. 2015, 2015:281.

[22] V. G. MAZ'JA, Sobolev spaces, Translated from the Russian by T. O. Shaposhnikova, Springer Series in Soviet Mathematics, Springer-Verlag, Berlin, 1985.

[23] E. Mitidieri, A simple approach to Hardy inequalities, (Russian) Mat. Zametki 67 (2000), no. 4, 563-572; translation in Math. Notes 67 (2000), no. 3-4, 479-486.

[24] A. Lieb, M. Loss, Analysis (second edition), Graduate Studies in Mathematics, 14, Amer. Math. Soc. Providence, RI, (2001), xxii+346 pp.

[25] F. RELlich, Halbbeschränkte Differentialoperatoren höherer Ordnung, (German) Proceedings of the International Congress of Mathematicians, 1954, Amsterdam, vol. III, pp. 243-250. Erven P. Noordhoff N. V., Groningen; North-Holland Publishing Co., Amsterdam, 1956.

[26] M. SAno, F. TAKAhashi, Improved Rellich type inequalities in $\mathbb{R}^{N}$, Springer Proceedings in Mathematics \& Statistics 176, in Geometric Properties for Parabolic and Elliptic PDE'sv, (2016), 241-255.

[27] M. Sano, F. TAKahashi, Scale invariance structures of the critical and the subcritical Hardy inequalities and their improvements, Cal. Var. PDEs, 56 (2017), no. 3, 56-69.

[28] M. Sano, F. TAKahashi, Some improvements of the Caffarelli-Kohn-Nirenberg type inequalities, Differential Integral Equations, to appear.

[29] M. SANO, F. TAKAHASHI, Sublinear eigenvalue problems with singular weights related to the critical Hardy inequality, Electron. J. Diff. Equ., Vol. 2016 (2016), no. 212, 1-12.

[30] F. TAKAHASHI, A simple proof of Hardy's inequality in a limiting case, Archiv der Math., 104 (2015), no. $1,77-82$.

[31] G. Talenti, Elliptic equations and rearrangements, Ann. Scuola Norm. Sup. Pisa Cl. Sci. (4) 3 (1976), no. 4, 697-718.

[32] T. PassalacQua, B. Ruf, Hardy-Sobolev inequalities for the biharmonic operator with remainder terms, J. Fixed Point Theory Appl. 15 (2014), no. 2, 405-431. 